\title{
Drosophila homologs of transcriptional mediator complex subunits are required for adult cell and segment identity specification
}

\author{
Muriel Boube, ${ }^{1}$ Christian Faucher, Laurent Joulia, David L. Cribbs, ${ }^{2}$ and Henri-Marc Bourbon \\ Centre de Biologie du Développement-CNRS, 31062 Toulouse CEDEX 04, France
}

\begin{abstract}
The origins of specificity in gene expression are a central concern in understanding developmental control. Mediator protein complexes regulate transcriptional initiation, acting as modular adaptors linking specific transcription factors to core RNA polymerase II. Here, we identified the Drosophila homologs of 23 human mediator genes and mutations of two, $A T R A P 240$ and of $A T R A P 80$ (the putative fly homolog of yeast SRB4). Clonal analysis indicates a general role for $A T R A P 80$ necessary for cell viability. The $d T R A P 240$ gene is also essential, but cells lacking its function are viable and proliferate normally. Clones reveal localized developmental activities including a sex comb cell identity function. This contrasts with the ubiquitous nuclear accumulation of dTRAP240 protein in imaginal discs. Synergistic genetic interactions support shared developmental cell and segment identity functions of dTRAP240 and dTRAP80, potentially within a common complex. Further, they identify the homeotic Sex combs reduced product, required for the same cell/tissue identities, as a functional partner of these mediator proteins.
\end{abstract}

[Key Words: Mediator; SRB; transcription; cell identity; segment identity; homeotic; development]

Received April 26, 2000; revised version accepted September 28, 2000.

Transcription initiation is a central and highly regulated step in eukaryotic gene expression that unites core RNA polymerase II (Pol II) with numerous other factors. These include Pol II-associated general transcription factors (TFIIA, TFIIB, TFIID, TFIIE, TFIIF, and TFIIH) and sequence-specific regulators bound to gene enhancers (for review, see Hampsey and Reinberg 1999; Malik and Roeder 2000). Work of the last 10 years has brought to light a new class of transcription factor complex, the mediator (MED). The first known mediator components, encoded by the yeast $S R B / M E D$ genes, were identified by dominant mutations suppressing the conditional lethality caused by a C-terminal domain (CTD) mutation of the Pol II large subunit (Thompson et al. 1993). The biochemically purified Srb proteins interact physically with core RNA Pol II in the form of large protein complexes (Thompson et al. 1993; Kornberg 1999).

More recently, mediator complexes have likewise been identified in mammalian cells where, as in budding

\footnotetext{
${ }^{1}$ Present address: Department of Molecular and Cellular Biology, CIDCSIC, c/ Jordi Girona 18-26, Barcelona 08034, Spain.

${ }^{2}$ Corresponding author.

E-MAIL cribbs@cict.fr; FAX 33-561-55-65-07.

Article and publication are at www.genesdev.org/cgi/doi/10.1101/ gad. 179800 .
}

yeast, they associate with the core Pol II to form a giant holoenzyme (Asturias et al. 1999). The mammalian MED complexes capable of stimulating basal transcription initiation in vitro contain $\sim 20$ subunits, including at least five proteins homologous to yeast Srb/MED proteins (Boyer et al. 1999; Gu et al. 1999; Ito et al. 1999; Naar et al. 1999; Rachez et al. 1999; Ryu et al. 1999). Several related complex forms that mediate transcription in vitro have been isolated through their physical contact with a spectrum of mammalian transcription factors. These include nuclear receptors for thyroid hormone (TRAP complex; Ito et al. 1999) and vitamin D3 (DRIP complex; Rachez et al. 1999), VP16, the p65 subunit of NF-кB, SREBP-1a (ARC; Naar et al. 1998, 1999), Sp1 (CRSP; Ryu et al. 1999), E1A (Boyer et al. 1999), and p53 (Ito et al. 1999). Alternative protocols have yielded related mammalian complexes (human or mouse mediator; Chao et al. 1996; Jiang et al. 1998; SMCC, Ito et al. 1999) or subcomplexes (negative regulator of activated transcription, NAT, associated with human Srb10/Cdk8; Sun et al. 1998). These related complexes are viewed as versatile interfaces that link specific transcription factors and the general Pol II machinery in a complex equilibrium (Lee et al. 1998). The MED complexes are most often considered transcriptional coactivators, and this property has been used as the basis of their biochemical 
Fly mediator genes in cell and segment identity

purification. Importantly, however, these complexes are not dedicated activators, and some forms have also been described as corepressors (Song and Carlson 1998; Sun et al. 1998).

A systematic examination of gene expression in yeast $s r b$ mutants has revealed distinct families of target genes of varying size and composition for different Srb subunits (Holstege et al. 1998). This functional diversity is likely to reflect a corresponding diversity of physically interacting regulatory transcription factors. MED complexes appear to integrate regulatory information from multiple transcription factors and relay that information to the core Pol II (for reviews, see Parvin and Young 1998; Hampsey and Reinberg 1999; Kornberg 1999; Malik and Roeder 2000). In support of this view, recent work demonstrates that human ARC complex can interact with two transcription factors and parlay this input into a synergistic transcriptional response (Naar et al. 1998, 1999|. In metazoans, the dynamic developmental process requires fine control of the gene expression program, presumably involving their MED complexes. However, for the moment little is yet known of their in vivo functions in development. The first known mutation of a metazoan MED subunit, in the sur2 locus of the nematode Caenorhabditis elegans, was isolated as a suppressor of activated Ras in vulval development (Singh and Han 1995). A MED complex has been identified in C. elegans and suggested to participate in regulation of developmental target genes (Kwon et al. 1999). Recently, gene inactivations have been described for two mouse subunits, Srb7 (Tudor et al. 1999) and TRAP220 (Ito et al. 2000). Murine Srb7 corresponds to a core MED subunit required for yeast cell viability and is apparently required for cell viability in the mouse embryo as well. The inactivation of TRAP220, a subunit implicated in ligand-dependent binding to thyroid hormone receptor, reveals diverse developmental defects in a variety of tissues.

A better understanding of the in vivo physiological roles of the MED complexes in metazoan development requires the continued genetic analysis of mutations of their subunits in model organisms. Toward this end, we have identified 23 Drosophila homologs of human MED subunits in the recently completed genomic sequence. We have cloned two of these and identified loss-of-function mutations for each. Both mutations are recessive lethal, revealing the functions of the genes as essential. However, the two show marked differences, as one is required for cell viability and the other is required for the integrity of the organism. These mutations have allowed us to identify discrete roles for MED protein functions in the control of adult segment and cell identities. Synergistic genetic interactions between the two putative MED subunits indicate a shared developmental function, potentially within a Drosophila complex. Their synergistic interactions with the homeotic Sex combs reduced ( $S c r$ ) locus further implicate the SCR homeodomain protein as a functional partner whose activity is modulated by MED protein activity during development.

\section{Results \\ Identification of 23 Drosophila homologs of human mediator proteins and cloning of pap/dTRAP240}

A new Drosophila gene, poils aux pattes (pap; cytological locus 78A1-3), was identified in a P-element screen for dominant genetic modifiers of cell identity functions of the homeotic loci Scr (Hox-A5/B5) and proboscipedia ( $p b$; Hox-A2/B2; Pattatucci et al. 1991; Cribbs et al. 1995). The $S c r$ selector gene confers prothoracic identity, while $p b$ alone induces maxillary identity. Together, the $S c r$ and $p b$ selectors show a combinatorial behavior leading to specification of the adult labial palps (mouthparts). Low-level ectopic expression of PB protein from an hsp70-pb mini-gene, the HSPB element, induces several dose-sensitive cell-identity phenotypes (Cribbs et al. 1995; Boube et al. 1997) that were used to screen for second-site dominant modifier mutations. Of 5000 new autosomal $P$ insertions tested, only one, in the pap locus, showed dose-sensitive enhancement of the distal sex comb (described below) induced by the HSPB element. Starting from the $P$-element molecular tag, a 50-kb pair interval encompassing the pap gene was cloned. Analysis of genomic and complementary DNA (cDNA) sequences indicates a transcription unit spanning at least $22 \mathrm{~kb}$ and generating $\mathrm{a} \sim 10-\mathrm{kb}$ mRNA (Fig. 1a). The exonic $P$ insertion resides upstream of the first in-frame ATG of an open reading frame (ORF) of 2618 amino acids (see Fig. 1a). Full reversion of lethality by mobilizing the $P$ element, and the rescue of lethality by a ubiquitin-cDNA construct (not shown), confirmed that this ORF corresponds to pap. The ORF encodes the unique Drosophila counterpart of TRAP240/ARC250, recently identified as a subunit of the human thyroid hormone receptor-associated protein (TRAP) or activator-recruited cofactor (ARC) protein complexes (Ito et al. 1999; Naar et al. 1999). The Drosophila PAP protein shows $27 \%$ overall identity $140 \%$ similarity) with human TRAP240 and $27 \%$ identity (39\% similarity) with its C. elegans counterpart. This conservation extends across the proteins but is highest in the $\mathrm{N}$ - and C-terminal regions (Fig. 1b). We therefore consider pap as the presumptive fly homolog of TRAP240.

The recent availability of the Drosophila genomic sequence (Adams et al. 2000) and the collection of corresponding cDNAs through the Berkeley Drosophila Genome Project (BDGP; Rubin et al. 2000) allowed us to identify a single putative Drosophila homolog for each of the 23 known human TRAP/ARC subunits (Fig. 2a; Materials and Methods). For simplicity, we will refer to these proteins and their genes in the text as TRAPs when more than one name exists for the same entity (Malik and Roeder 2000). While the existence of a biochemical entity remains to be demonstrated, the observed structural conservation of such a large number of $M E D$ genes provides clear circumstantial evidence for the existence of a fly mediator complex (dMED) similar to the purified complexes from worms (Kwon et al. 1999) and mammals (above). 
Boube et al.

$\mathbf{a}$ pap/dTRAP240 locus (78A1-3)

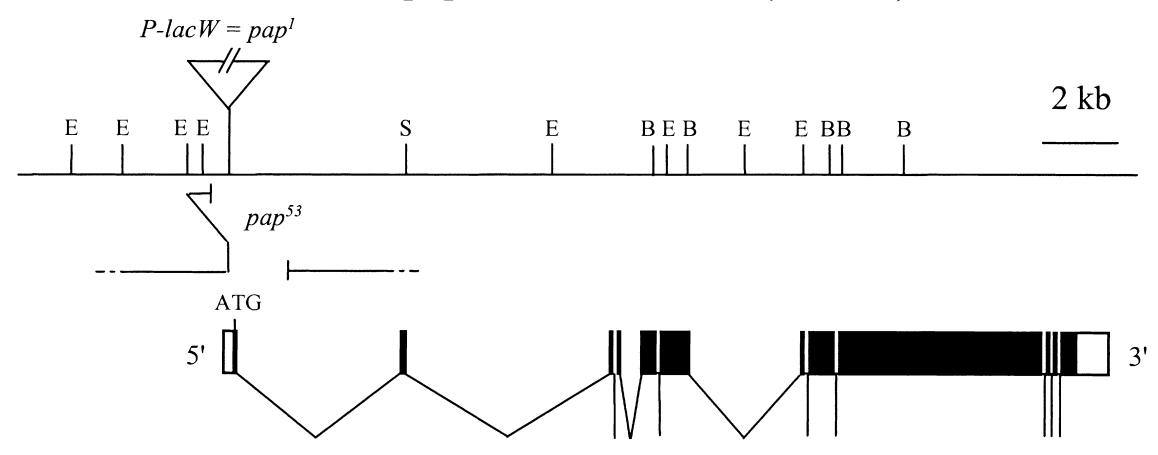

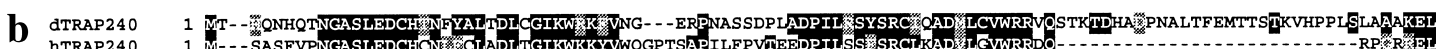

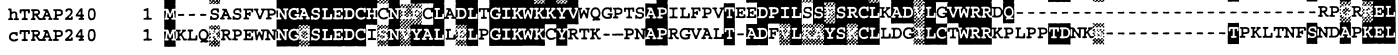

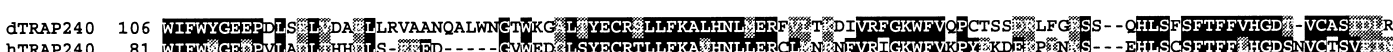

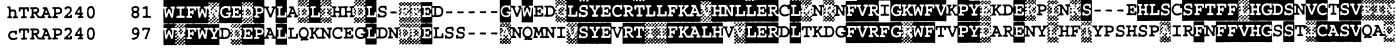

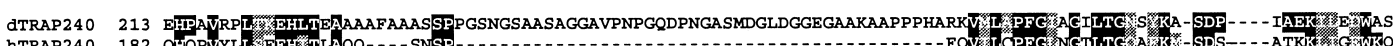

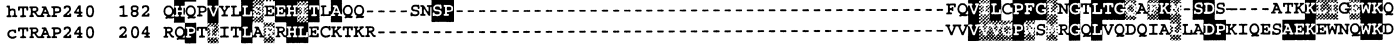

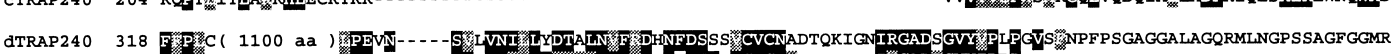

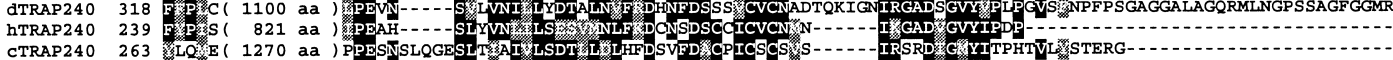
ATRAP240 1512 MISAFGGSPASASMPGAGSGHGHGPNGGSNSS勧CTPPS"

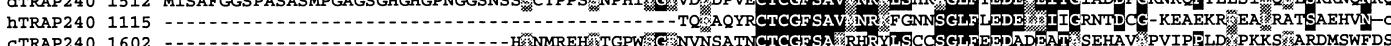
C1RAP240 160210

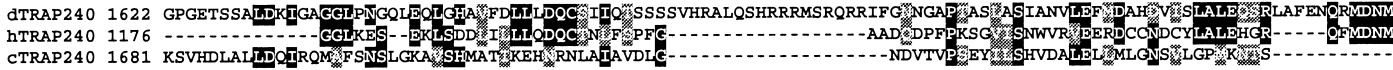

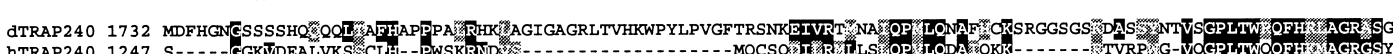

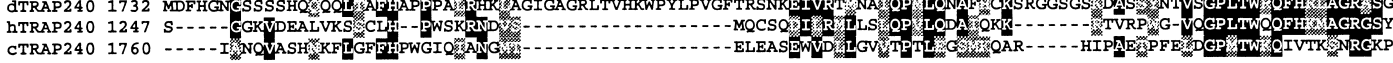

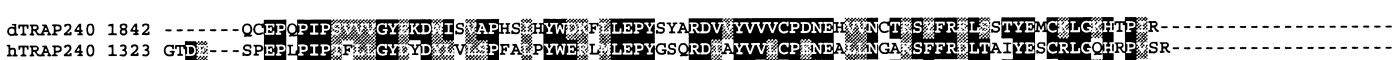

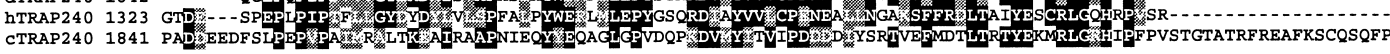

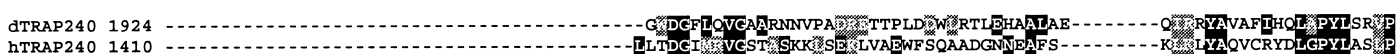

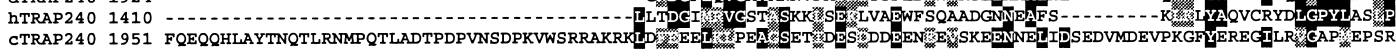
ATRAP240 1983 NDT TLLWPP.

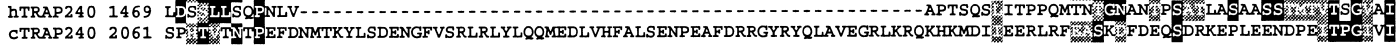

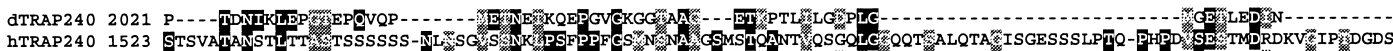

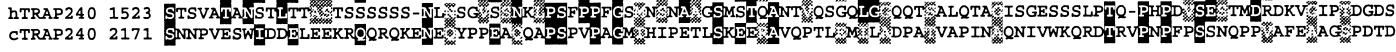

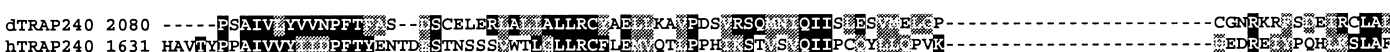

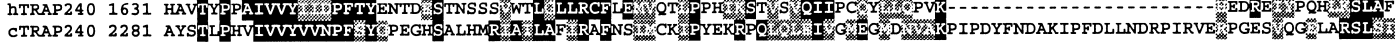

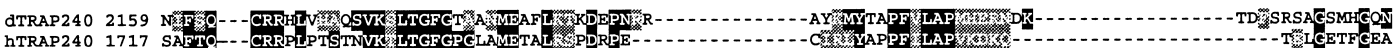

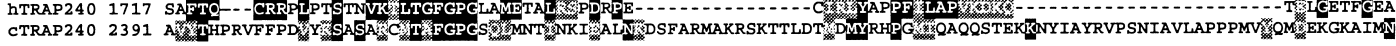

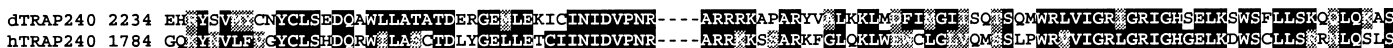

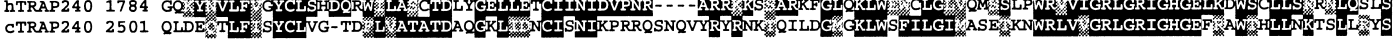

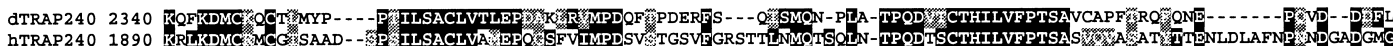

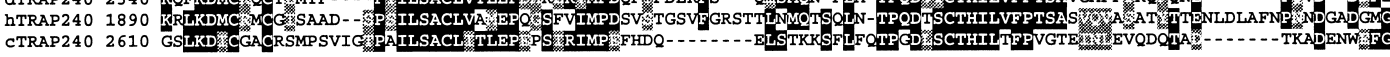

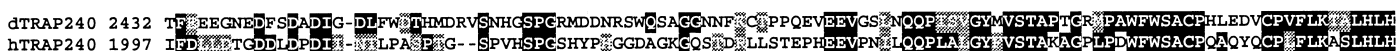

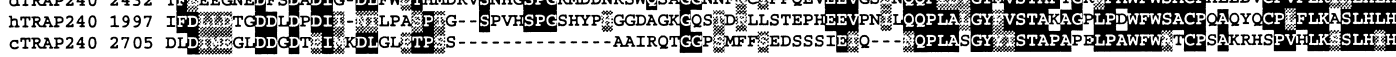

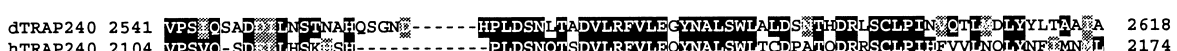

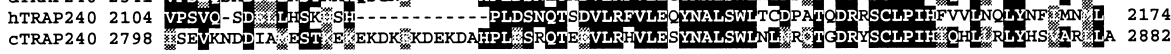

Figure 1. pap encodes the fly homolog of the human transcriptional mediator subunit TRAP240. (a) Molecular organization of the pap/dTRAP240 region (cytological position 78A1-3, chromosome 3L). The position of the recessive lethal P-lacW element insertion allele pap $^{1}$ is shown with the map of this region. (E) EcoRI; (B) BamHI; (S) SalI. The pap ${ }^{53}$ allele deletes the entire coding portion of exon 1 (amino acids 1-23). The transcription unit is shown with boxes representing exons; ATG is the presumptive initiator codon, filled boxes indicate protein coding region, and broken lines indicate introns (the precise site of transcription initiation is not known). (b) Protein sequence comparisons of TRAP240 homologs from Drosophila (d), human (h), and Caenorhabditis elegans (c). Because of the length of the proteins, only the most conserved regions (the $\mathrm{N}$ and $\mathrm{C}$ termini) are shown. Identical amino acids are shaded in black and conservative changes in gray. The Genbank accession numbers for the fly and human proteins are AF227214 and AF117754, respectively. The putative C. elegans homolog is derived from EMBL accession number Q93442, with aligned sequence beginning at a conserved methionine. 
a

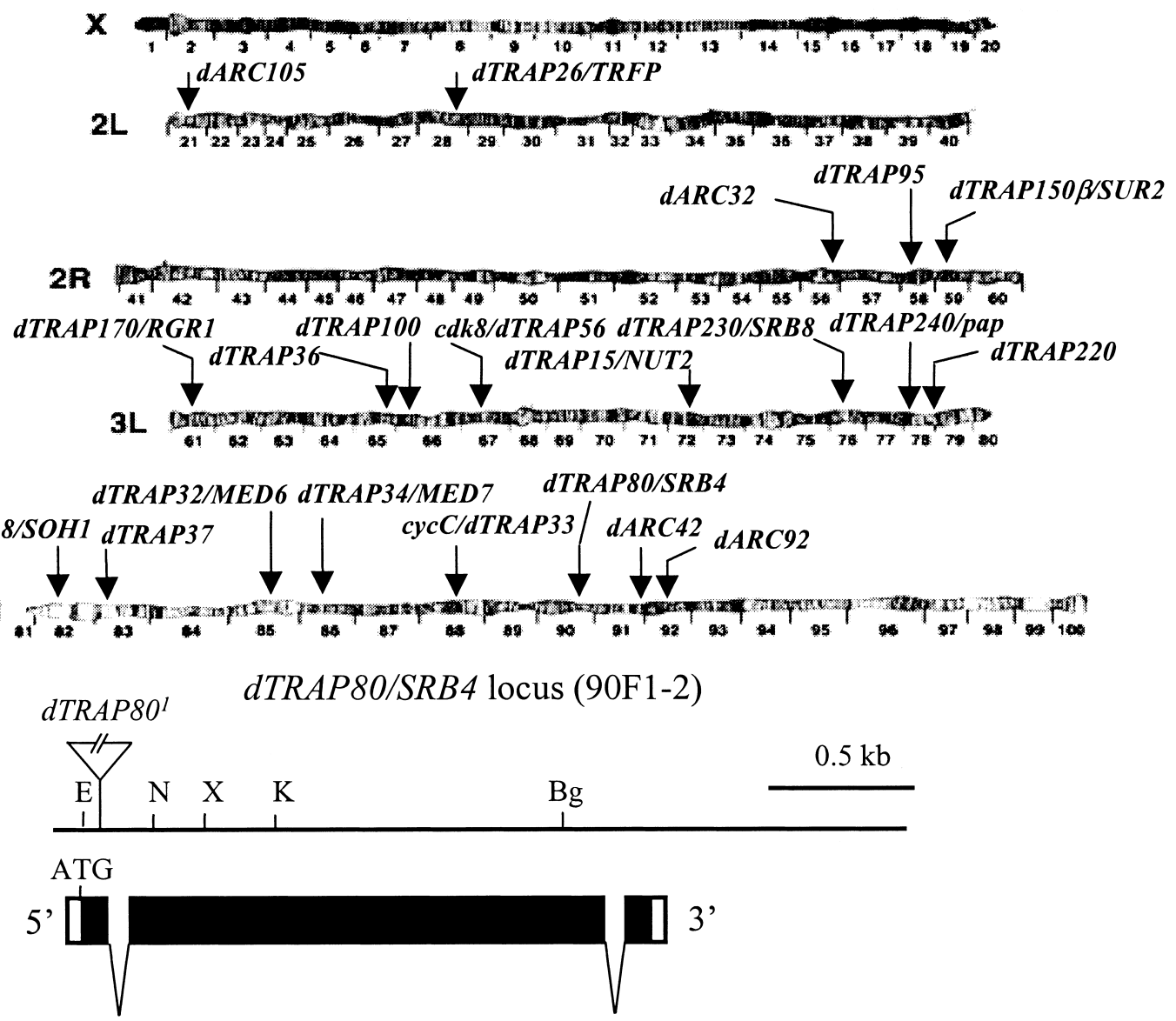

c

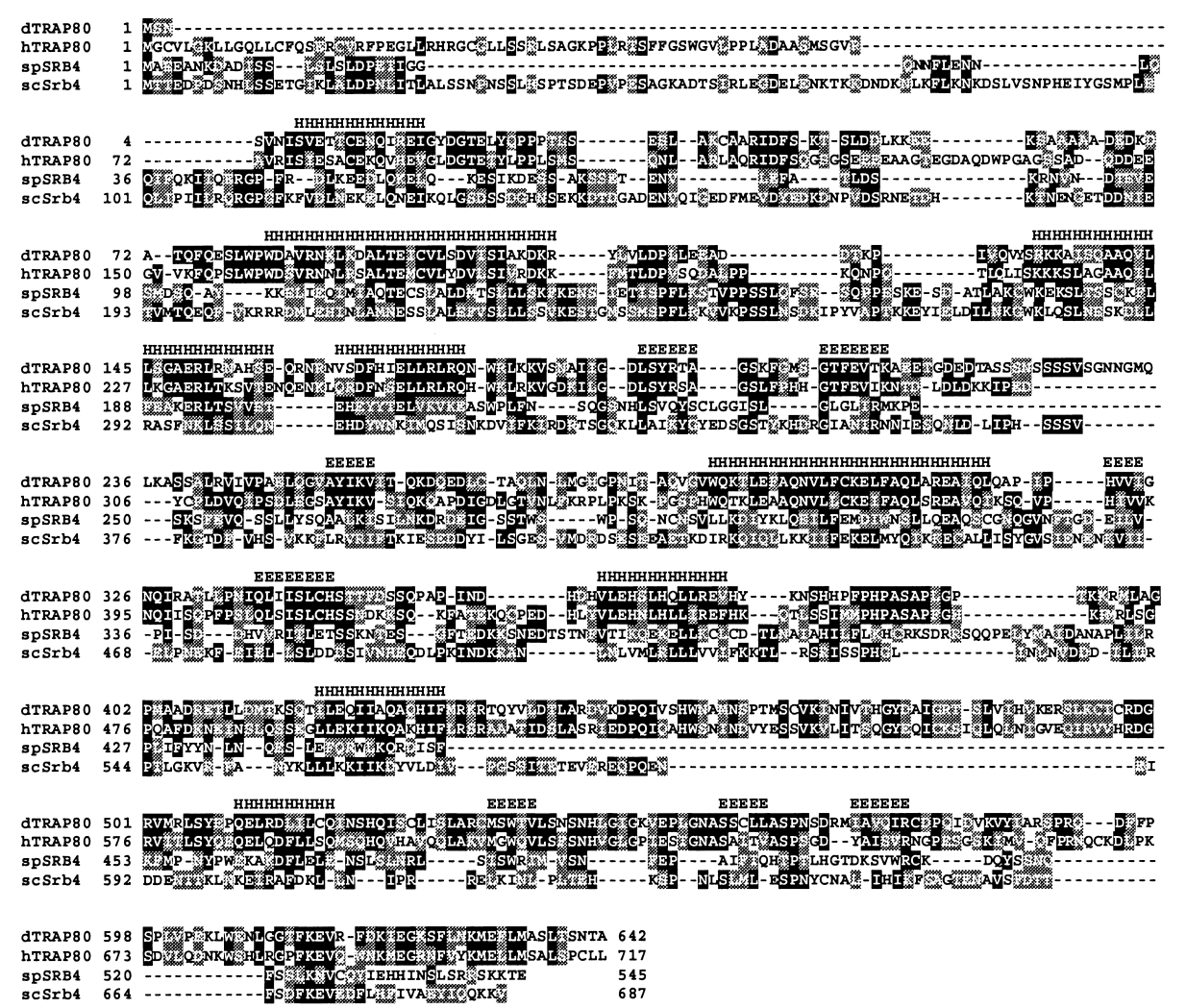

Figure 2. (Legend on following page) 
pap and dTRAP80 encode putative mediator subunits required for development

Among the new $d M E D$ genes identified was $d T R A P 80$ at cytological position 90F1-2 on chromosome 3R (Fig. 2b). It encodes a predicted dTRAP80 protein of 642 amino acids exhibiting $40 \%$ identity (59\% similarity) to its human counterpart. The majority of putative Drosophila $M E D$ genes, including pap, appear to lack a homolog in the complete Saccharomyces cerevisiae genome sequence. S. cerevisiae SRB4 encodes a core component of the Srb mediator complex required for the expression of virtually all yeast genes (Holstege et al. 1998). The gene was identified by dominant mutations that directly suppress a Pol II CTD mutation (Thompson et al. 1993). For the dTRAP80 protein, low but potentially significant overall structural conservation (16\% identity, $48 \%$ similarity) was detected with Srb4 proteins from the MED complexes of the yeast $S$. cerevisiae and Schizosaccharomyces pombe (Thompson et al. 1993; Spahr et al. 2000). The overall identity between these two yeast Srb4 proteins is only $25 \%$ (60\% similarity), with conservation most pronounced in a region with predicted $\alpha$-helical character between amino acids 214-313 of S. cerevisiae Srb4 (40\% identity, $72 \%$ similarity; see Fig. 2c). Both primary sequence and predicted helical character are conserved within this interval in metazoan TRAP80 moieties, attaining $34 \%$ identity between human TRAP80 and $S$. pombe SRB4. The corresponding sequences appear unique in the budding yeast and Drosophila genomes, arguing against a novel reiterated domain. Thus, despite the low level of overall identity, these observations are good evidence for homology of the metazoan TRAP80 genes with yeast SRB4.

One lethal $P$-insertion mutation from the BDGP collection (Spradling et al. 1999) is situated within the dTRAP80 coding sequence. This insertion, $d T R A P 80^{1}$, is located downstream of the apparent initiator ATG within the same exon (Fig. 2b). The cloning and the identification of mutations in these two putative Drosophila $M E D$ genes allowed us to initiate an in vivo assessment of their physiological roles in normal development. dTRAP $80^{1}$ mutants die as second-instar larvae with no obvious cuticular defect. The initial pap $^{1} P$-element insertion and most derived imprecise excisions (including the molecular null allele $\mathrm{pap}^{53}$; see Fig. 1a) are recessive embryonic lethals. pap ${ }^{-}$embryos appear normal apart from discrete cuticular defects of the embryonic mouthparts. Thus, both functions are essential for viability, and pap is detectably required for normal embryonic development. Ubiquitous accumulation of pap and dTRAP80 mRNA was observed by in situ hybridization in embryos of all stages and in larval imaginal discs (not shown). The presence of mRNA in early embryos further suggests that a maternal contribution partially compensates for the absence of zygotic expression for both pap and $\operatorname{dTRAP} 80$.

\section{dTRAP80 loss of function is cell lethal}

The prototypical Srb4 protein is required for transcription of nearly all Pol II-dependent promoters in yeast (Holstege et al. 1998). If $d T R A P 80$ encodes the functional homolog of Srb4, it is predicted to participate in all aspects of mediator function, and the $d T R A P 80^{-}$condition should be cell lethal. The survival of $d T R A P 80^{-}$embryos to second-instar larvae (above) suggested that maternally contributed $d T R A P 80$ mRNA suffices for embryonic survival. To test the consequences of removing dTRAP80 while minimizing the complication of maternal contribution, we employed mitotic recombination. From heterozygous mother cells, twin clones of daughter cells homozygous for each of the two chromosome arms

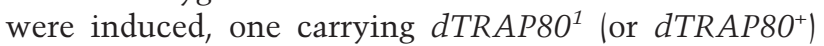
and the other its wild-type homolog (plus the associated cuticular markers Stubble $[S b]$ and ebony $[e])$. The dTRAP80 ${ }^{+/+}$or $d T R A P 80^{-/-}$cells of interest were identified by their bristle shape $\left(S b^{+}\right)$. Where $d T R A P 80^{+}$ yielded 150 clones, none were observed with dTRAP80 ${ }^{-1-}$ for an equivalent sample size. We conclude that $A T R A P 80$ is required for cell viability in the adult epidermis. This result provides independent support for a general cellular role of $d T R A P 80$ consistent with the sequence-based interpretation that it encodes a fly Srb4.

pap encodes a ubiquitous nuclear protein with localized developmental functions

To examine the distribution and cellular localization of PAP protein, a polyclonal antiserum was generated against its N-terminal region, then enriched by affinity chromatography with the same PAP protein fragment

Figure 2. Identification and genomic localization of Drosophila melanogaster homologs to known human MED genes. These include dTRAP80, an apparent metazoan homolog of yeast SRB4. (a) Cytological positions of 21 predicted $d M E D$ loci are shown. These genes have been annotated by the Genome Annotation Database of Drosophila (GadFly; http//www.fruitfly.org/annot/) and are listed in Materials and Methods. Homology searches identified $d T R A P 80$ as the apparent metazoan homolog of yeast SRB4 (see $c$ ). (b) Genomic organization of the $d T R A P 80$ locus (cytological interval 90F1-2 of chromosome 3R) is shown. The $d T R A P 80$ region map, (E) EcoRI; (N) NotI; (K) KpnI; (Bg) BgIII, indicates the position of the recessive lethal $d T R A P 80^{1}$ P-element insertion, previously referred to as 1(3)s2956 (Spradling et al. 1999). The exon-intron organization is shown beneath with filled boxes for protein coding region and broken lines indicating introns. (c) Aligned protein sequences of TRAP80/Srb4 homologs from D. melanogaster (d), human (h), Schizosaccharomyces pombe (sp), and Saccharomyces cerevisiae (sc). Genbank accession numbers are AF244916, AF117657, CAB10081, and L12026, respectively. A Caenorhabditis elegans TRAP80 homolog was also detected (not shown; Genbank accession number CAB76740). Identical amino acids are shaded in black, and conservative changes (Taylor 1986) in gray. Positions of aligned amino acids predicted to adopt helical $(\mathrm{H})$ or extended $(\mathrm{E})$ secondary conformations (http//www.ibcp.fr/predict.html) are indicated above the alignment. 
(Materials and Methods). The specificity of the antisera was confirmed by immunostaining of embryos and of imaginal discs overexpressing PAP in the posterior compartment of each segment (engrailed-GAL4/UAS-pap) or of discs harboring mitotic clones of $\mathrm{pap}^{53}$ cells (Materials and Methods). In overexpression experiments, strong staining was limited to posterior cells, whereas in clones of $\mathrm{pap}^{53}$ cells, the signal was no longer detected (not shown). Immunostaining experiments with this specific anti-PAP serum show that pap mRNA is translated throughout the imaginal tissues, as predicted by the mRNA distributions. PAP protein accumulation is predominantly nuclear (Fig.3a,b,d,e), in agreement with a role in the general transcription machinery.

To examine functional requirements for the essential pap gene in adult development, we generated mitotic clones of mutant cells. In marked contrast to dTRAP8O (above), clones were obtained showing that normal pap function is not required for cell viability. Clones induced during larval development (Fig. 3) led to distinct consequences in different tissues. First, adult Drosophila melanogaster males normally align a single row of specialized bristles, the sex comb teeth, on the first tarsal segment of the prothoracic (T1) leg. These sex comb teeth are not found elsewhere. In contrast, clones of pap mutant cells situated in the distal second tarsal segment differentiated as ectopic sex comb teeth (Fig. 3c). Normal pap function thus opposes sex comb cell fate in this position. This role appears cell autonomous, as all observed ectopic sex comb teeth were mutant for pap (see Fig. 3c). In contrast, clones within the normal sex comb or bordering it did not affect the number of cells adopting this fate (Fig. 3c). Second, clones localized elsewhere in the $\mathrm{T} 1 \mathrm{leg}$, or at any position in the $\mathrm{T} 2$ and $\mathrm{T} 3 \mathrm{legs}$, were without effect (not shown). Third, clones in the maxillary palps are associated with malformations. Finally, large clones in the wing blade, the notum, or the antennae led to apparently normal pattern. Therefore in contrast with ubiquitous accumulation of PAP in epidermal cells, pap function is required for normal development in only a subset of those cells. Taken together, these data strongly suggest that developmental pap activity may be regulated according to the tissue and cell, being required for some identities but dispensable for others.

Previous biochemical characterization identified human TRAP240 as a component of the ubiquitous MED complexes and, hence, of the general transcription machinery. As such, pap/dTRAP240 mutations might have
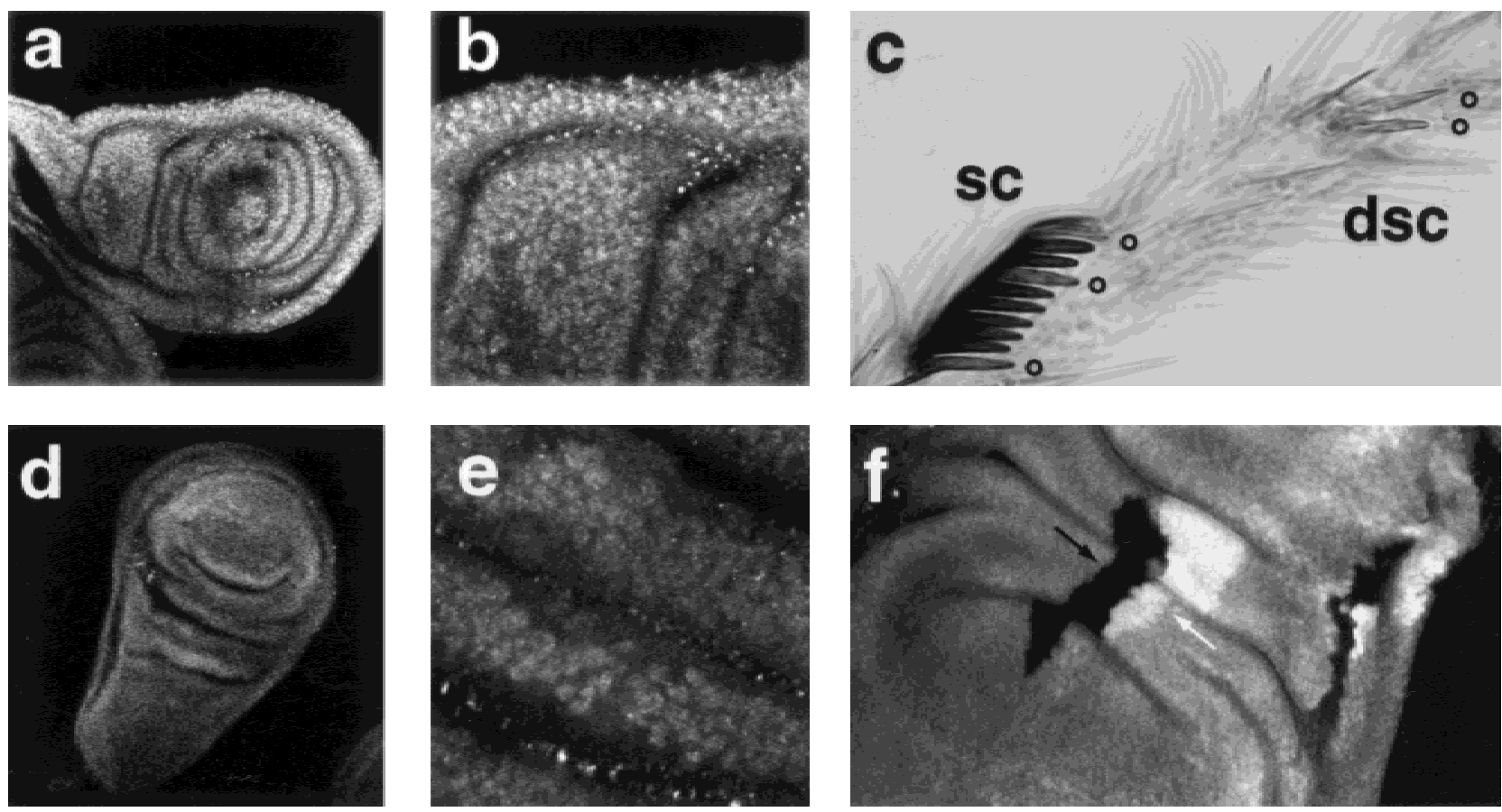

Figure 3. Expression and activity of the pap/dTRAP240 locus. $(a, b, d, e)$ Localization of PAP in imaginal discs from third-instar larvae using a monospecific rabbit polyclonal serum. Shown are a prothoracic leg imaginal disc $(a)$ with enlargement $(b)$, and a wing imaginal disc $(d)$ with enlargement $(e)$. PAP protein is expressed in most or all imaginal cells $(a, d)$ and accumulates preferentially in the nucleus $(b, e)$. Mutant clones at the distal extremity of the second tarsal segment in the T1 legs $(c)$ exhibited an ectopic distal sex comb (dsc). Ectopic sex comb teeth (small circles) are yellow $\left(\mathrm{pap}^{-}\right)$. A maximum of three ectopic teeth were observed in any pap clone. Within the normally placed sex comb (sc) at the distal end of the first tarsal segment of this sample, three mutant cells (marked with circles) are interspersed with $\mathrm{pap}^{+}$cells. This sex comb is of normal size. Hence, the pap ${ }^{-}$condition affected neither sex comb cell differentiation nor rotation for alignment. $(f)$ Twin spot clonal analysis of pap function in imaginal disc development. Adjacent (twin) clones of $\mathrm{pap}^{-/-}$(no GFP; black arrow) and $\mathrm{pap}^{+/+}$cells (two copies of ubiquitin-GFP; white arrow) are identified by expression of the cell-autonomous GFP marker in a third-instar larval wing imaginal disc. Note the similar sizes and distributions of the wild-type and mutant cells. 
adverse consequences for cell physiology and growth. Consequently, the discrete changes in cell identity observed could reflect a spurious fragilization of the cell rather than a concrete developmental role for pap in cell specification. To address this concern, clonal analysis was performed (Materials and Methods and above) using a green fluorescent protein (GFP) cell marker to examine the proliferation and distribution of mutant cells relative to wild type. Clones of cells homozygous for $p^{2} p^{53}$ and the reciprocal wild-type product were induced in first- or second-instar larvae, then compared in imaginal discs of late third-instar larvae. An example is shown in Figure $3 \mathrm{f}$, where twin clones are observed in a wing imaginal disc. Adjacent clones of mutant cells (black) are as large as their wild-type counterparts (white) and similarly distributed relative to the axes of the imaginal disc. Similar results were obtained in other imaginal tissues, including the labial and $\mathrm{T} 1 \mathrm{leg}$ discs (examples in Fig. 4 below). These results reinforce the conclusion that the distal sex comb cell identity transformation observed in pap mutant clones reflects a normal, localized function of this gene in this position and not an indirect consequence of an impaired general transcription machinery function.

pap and dTRAP80 interact synergistically with the homeotic Sex combs reduced locus in cell and segment identity specification

The ectopic distal sex comb induced by pap clones is a readily visible cell identity marker that reflects normal pap function. Ectopic distal sex comb teeth are induced in appropriately positioned cells lacking any pap function (above). This phenotype is also observed at low frequency with certain Scr and $p b$ gain-of-function alleles $\left(S c r^{S c x P}\right.$ and hsp70-pb [HSPB] mutations; Pattatucci et al. 1991; Cribbs et al. 1995). This effect of the $S c r^{S c x P}$ allele is enhanced in pap heterozygotes (Table 1). Functions of the homeodomain transcription factor SCR specify prothoracic identity, including sex comb cell fate. The in-

Table 1. Scr and dMED in distal sex comb (DSC) cell identity

\begin{tabular}{|c|c|c|c|}
\hline Genotype & $\begin{array}{l}\text { Percentage } \\
\text { legs with } \\
\text { DSC }\end{array}$ & $\begin{array}{l}\text { Mean } \\
\text { DSC } \\
\text { teeth } \\
\text { per leg }\end{array}$ & $\begin{array}{l}\text { Percentage } \\
\text { of legs with } \\
\text { three or more } \\
\text { DSC teeth }\end{array}$ \\
\hline $\operatorname{pap}^{53} /+$ & $0(n=200)$ & 0 & 0 \\
\hline$S c r^{S c x P}$ & $3(n=182)$ & 0.04 & 0 \\
\hline$S c r^{S c x P} / p a p^{53}$ & $15(n=102)$ & 0.15 & 0 \\
\hline HSPB & $6(n=344)$ & 0.06 & 0 \\
\hline $\mathrm{HSPB} / \mathrm{Scr}^{4}$ & $0(n=252)$ & 0 & 0 \\
\hline $\mathrm{HSPB} /$ pap $^{53}$ & $54(n=206)$ & 0.54 & 1 \\
\hline $\mathrm{HSPB} / \mathrm{pap}^{53} \mathrm{Scr}^{4}$ & $0(n=171)$ & 0 & 0 \\
\hline dTRAP80 $1++$ & $0(n=200)$ & 0 & 0 \\
\hline pap $^{53}$ dTRAP80 $/++$ & $10(n=372)$ & 0.1 & 0 \\
\hline$S c r^{S c x P} / d T R A P 80^{1}$ & $9(n=140)$ & 0.1 & 0 \\
\hline$S c r^{S c x P} / p a p^{53}$ dTRAP8O ${ }^{1}$ & $34(n=150)$ & 0.4 & 10 \\
\hline
\end{tabular}

duction of distal sex combs by HSPB also depends on $S c r$ activity, as it is no longer detected in Scr heterozygotes (see Table 1). Ectopic sex comb differentiation is enhanced in $\mathrm{HSPB} /$ pap $^{53}$ heterozygotes, but this effect is abolished in Scr heterozygotes (Table 1). These observations of dose-sensitive interactions indicate a synergistic functional link between $S c r$ and pap in this cell identity specification. pap and dTRAP80 both encode the sole detected fly homologs to human proteins identified by their presence in the MED complex. If the enhancement of the distal sex comb phenotype in pap heterozygotes is caused by limiting mediator function, double heterozygotes with $d T R A P 80$ should aggravate this condition. We therefore tested whether $A T R A P 80$ acts together with pap in this cell identity decision. The loss-of-function allele $d T R A P 80^{1}$ is fully recessive, as for $p a p^{53}$. In contrast, $10 \%$ of $\mathrm{pap}^{53}+/+d T R A P 80^{1}$ males possess an ectopic distal sex comb tooth, revealing a cooperative function in these cells (Table 1). Synergistic enhancement of the ectopic sex comb caused by $S c r^{S c x P}$ is likewise observed in double heterozygotes (Table 1). These functional data indicate a shared function of PAP and dTRAP80, again suggesting the existence of a Drosophila mediator complex. They further suggest that at least one common function of PAP and dTRAP80 acts to antagonize $S c r$ activity in distal sex comb differentiation.

Apart from the prothorax, normal Scr activity is also required for development of the adult labial palps, where it acts in a combinatorial fashion with the homeotic $p b$ gene (Cribbs et al. 1995; Percival-Smith et al. 1997). This led us to examine the effects of pap and $A T R A P 80 \mathrm{mu}-$ tations on adult mouthparts formation. The wild-type labium is typified by the presence of pseudotracheal rows used for drinking and the absence of a segmental appendage. The hypomorphic $p b^{4} / p b^{5}$ genotype leads to a transformation of distal labium to antennal arista (Fig. 4a), with a concomitant reduction of the pseudotracheae. In this sensitized context, changes in relative $S c r$ activity can be readily detected. Reduced pap or ATRAP80 activity in heterozygotes enhances the labial-to-leg transformation, as seen by the appearance of leg-specific cell types: sex comb teeth in males, bracted bristles, and terminal claws (Table 2). In pap dTRAP80, double heterozygotes leg structures often entirely replace labial pseudotracheae (Fig. 4c). As in the leg, this effect of pap and dTRAP80 mutants on labial development is synergistic (see Table 2). These data provide further support for a shared role of PAP and dTRAP80 proteins opposed, in this case, to the leg-forming activity of Scr in the labial tissue.

\section{pap and the homeotic selector locus Scr act in parallel}

The observed effects of pap and $\operatorname{dTRAP} 80$ mutations in the $\mathrm{T} 1$ legs and labium may most simply be rationalized as consequences of increased Scr activity. This could result from augmented regulatory activity of SCR protein toward Scr target genes. Alternatively, it might reflect higher Scr gene expression with greater quantities of SCR protein. To distinguish between these two possibili- 

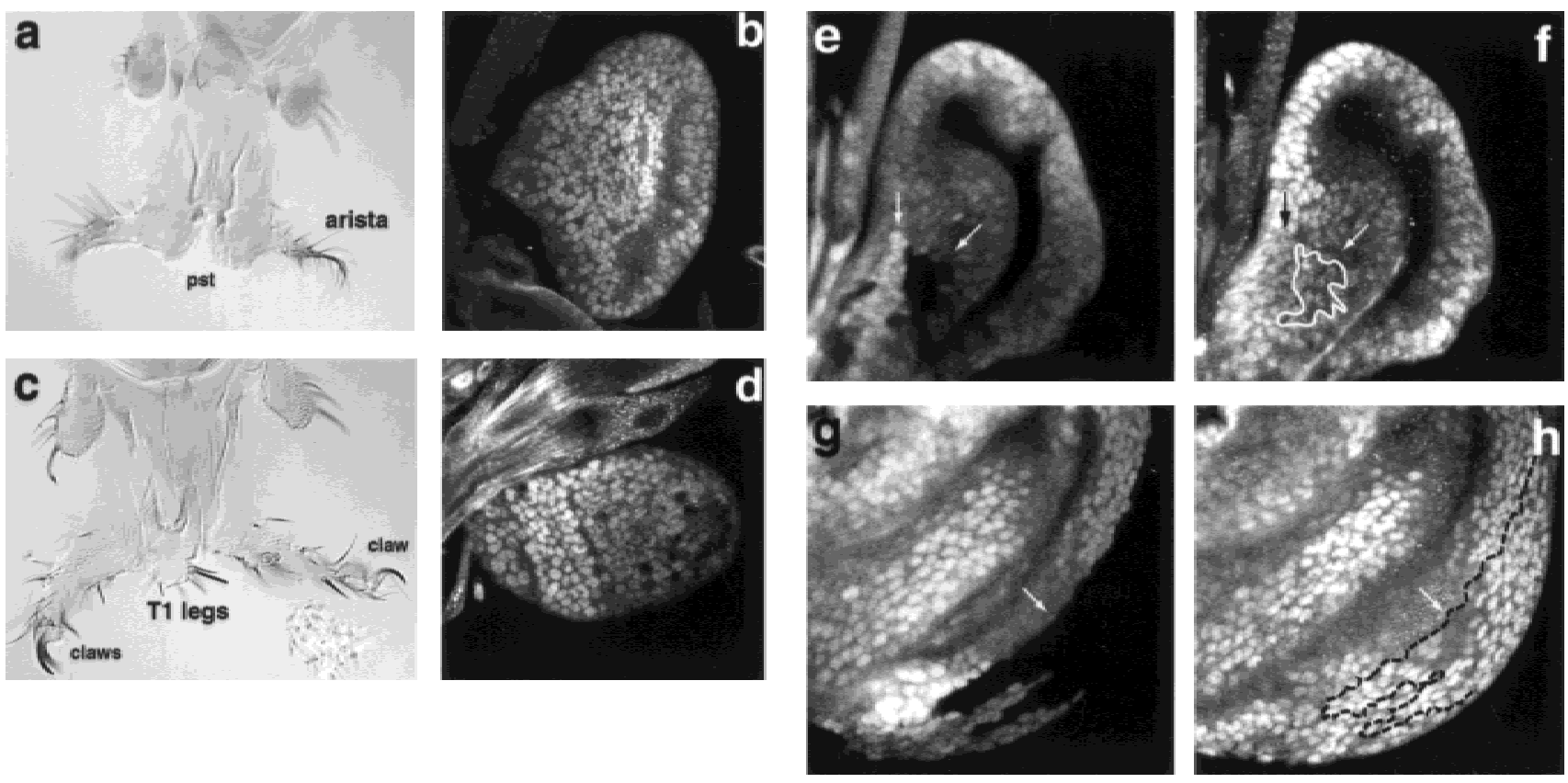

Figure 4. pap and $d T R A P 80$ functions contribute to segmental identity specification. Labial palp identity is determined by the joint action of $S c r$ and $p b .(a, c)$ Loss-of-function mutations of pap/dTRAP240 and dTRAP80 affect labial palp development, favoring T1 leg development. (a) The mutant combination $p b^{4} / p b^{5}$ (where $p b^{5}$ is a protein-null allele and $p b^{4}$ is a hypomorph leading to a C-terminally truncated PB protein) transforms distal labial palps to antennal aristae (arista), whereas the proximal labium retains normal pseudotracheal rows (pst). (c) The $p a p^{1}$ and $d T R A P 80^{1}$ mutations are recessive in this tissue but enhance the $p b^{4 /} p b^{5}$ mutant phenotype. Shown are mouthparts of a $p b^{4} / p a p^{1} p b^{5} d T R A P 80^{1}$ adult female. Morphology is altered compared to $a_{\text {; }}$ pseudotracheae are replaced by apparent leg structures, and claws replace the aristae. (b, $d) S c r$ acts with $p b$ in the labium to promote labial (or antennal/labial) identity but transforms the labium to distal T1 leg in the absence of $p b$. Accumulation of SCR protein was examined in imaginal labial disc cells of $p b^{4} / p b^{5}$ mutants, giving rise to mixed antennal/labial identities $(b)$ or $p b^{4} / p a p^{1} p b^{5} d T R A P 80^{1}$ mutants that give predominantly T1 leg tissue $(d)$. Nuclear SCR protein was detected throughout the discs in both cases, as it was for wild type. SCR accumulation is not increased and, indeed, may be somewhat reduced (cf. $b$ and $d$ ), in the discs giving rise to T1 legs. (e- $h) S c r$ expression examined in clones of pap $^{53}$ homozygous cells. (e) Shown is a labial imaginal disc harboring a twin spot clone of $+/+($ white, vertical arrow) or of $\mathrm{pap}^{53} / \mathrm{pap}^{53}$ cells (black, diagonal arrow). (f) Expression of the SCR homeodomain protein is seen in the same labial disc. The clone is outlined and indicated by the diagonal arrow. SCR accumulation is unaltered in the mutant cells. $(g)$ This T1 leg imaginal disc harbors a prominent clone of $\mathrm{pap}^{53} / \mathrm{pap}^{53}$ cells (black, limit indicated by arrow). (h) SCR protein accumulation in the same T1 disc. The clone is outlined and its border indicated by the arrow. SCR accumulation is unchanged in the pap cells.

ties, we examined SCR homeoprotein accumulation by indirect immunofluorescence in $p b^{4} / p b^{5}$ labial imaginal discs that give rise to mixed labial/antennal or $p b^{4} / p a p^{1}$ $p b^{5}$ dTRAP80 ${ }^{1}$, yielding T1 leg identities. Nuclear SCR protein does not detectably increase with the transition to T1 leg identity (Fig. 4, cf. b and d). These data indicate that PAP and dTRAP80, acting in parallel or downstream of Scr, negatively modulate SCR protein function in labial tissue.

The above experiments were performed in heterozygotes for pap and dTRAP80. In the sensitized genetic context employed, slight but functionally important changes in SCR accumulation could potentially pass undetected in this test. We therefore tested the molecular epistatic relations between pap and $S c r$ in homozygous mutant cells to determine whether Scr gene expression depends on normal pap function and vice versa. Both Scr and pap are normally expressed throughout the labial and T1 leg imaginal discs, and both confer detectable phenotypes there as described above. Mitotic clones of homozygous $\mathrm{pap}^{-/-}$or $\mathrm{Scr}^{-/-}$cells were induced in first- and second-instar larvae and identified in mature thirdinstar imaginal discs by the cell autonomous GFP marker and by the accumulation of SCR or PAP proteins examined in these cells of known genotype. Representative results for SCR protein accumulation are shown for pap $^{-}$clones in the labial (Fig. 4e,f) and T1 leg (Fig. 4g,h) imaginal discs. No change in SCR accumulation was detected in $\mathrm{pap}^{-/-}$cells compared with neighboring wild-

Table 2. dMED mutations and adult labial segment development

\begin{tabular}{lcc}
\hline Genotype & $\begin{array}{c}\text { Sex comb } \\
\text { teeth }(\%)\end{array}$ & $\begin{array}{c}\text { Claws } \\
(\%)\end{array}$ \\
\hline$p b^{4} / p b^{5}$ & 6 & 0 \\
$p b^{4} / p b^{5} \mathrm{pap}^{1}$ & 18 & 41 \\
$p b^{4} / p b^{5}$ dTRAP8O & 1 & 1 \\
$p b^{4} / p b^{5}$ pap $^{1}$ dTRAP8O & 53 & 62 \\
\hline
\end{tabular}

Note. Counts $(n>100$ in all cases) refer to half labia (derived from a single imaginal disc). 
type cells in either tissue. These results obtained in homozygous pap ${ }^{-}$cells confirm that Scr gene expression, as measured by accumulation of the nuclear homeodomain protein, is not detectably affected by altered pap function in these tissues. Conversely, PAP protein accumulation was unchanged in $\mathrm{Scr}^{-/-}$cells (not shown), indicating that pap transcription is likewise independent of Scr function in these tissues. These reciprocal experiments, coupled with the results in heterozygotes described above, provide molecular evidence that the pap and dTRAP80 loci act in parallel with homeotic Scr function in distal sex comb and labial identity specification.

\section{Discussion}

The MED complexes are believed to serve as adapting interfaces between regulatory proteins bound to specific DNA sequences and the RNA Pol II that executes this input. Overall complex architecture and physical interactions with RNA Pol II appear relatively unchanged between yeast and mammals (Asturias et al. 1999). In this work, we found a single fly homolog for each of 23 known human MED proteins. The presence of so many detectable fly homologs conforms with the observed conservation of overall complex structure between yeast and mammals and clearly supports the existence of a fly complex. The synergistic interactions detected in tests of double heterozygous mutant combinations for pap and dTRAP80 loss-of-function mutations revealed a shared developmental function. The most direct interpretation incorporating these two complementary lines of evidence is that these proteins act in vivo within the shared confines of a fly mediator complex, similar to those detected in worms and mammals.

Whether pap and $d T R A P 80$ act within a mediator complex remains unproven, and elucidating this point must await the biochemical demonstration of a fly complex containing both proteins. Still, the presence of so many recognizable homologs clearly suggests the existence of a metazoan complex, leading us to consider here the developmental and evolutionary implications of this working hypothesis.

The metazoan complex is likely to act as a motor force for evolving genetic programs: overall complex architecture might reflect constraints imposed by interaction with RNA Pol II, while even limited modifications of subunit sequences could alter contacts with specific transcription factors that amplify the modification. Speciation and morphological divergence reflect changes in gene expression programs. Clarifying how these MED protein functions modulate transcription should shed light on the constraints limiting or favoring evolutionary change. One of the two Drosophila genes isolated here, dTRAP80, encodes the apparent fly homolog of the prototypical yeast complex member Srb4. In contrast, pap/ dTRAP240 does not possess a detectable yeast equivalent. One intriguing possibility is that the pap gene may reflect a metazoan-specific MED protein, conceivably replacing a yeast protein of analogous function. The overall number of protein components of yeast and mammals appears similar, and some subunits of the yeast Srb complex are present in human MED as well. However, others such as pap are detectable in nematodes, flies, and mammals but apparently absent in yeast. It is not yet clear whether truly species-specific MED subunits exist. Still, the emergence or exchange of additional metazoan-specific subunits, as well as mixing and matching among various modular subcomplexes such as those detected in yeast (Lee and Kim 1998; Lee et al. 1999), may have been an important element contributing to the diversification of genetic programs in complex organisms.

Ample evidence indicates that yeast Srb complex subunits have diversified functions (Holstege et al. 1998). Some subunits such as Srb4 are required for transcription of nearly all yeast genes, whereas others are specific for discrete, largely nonoverlapping gene subsets. Information concerning the in vivo physiological roles of the metazoan MED complexes and their subunits remains sparse. A knock-out mutation of the ubiquitously expressed mouse Srb7 gene suggests a requirement for cell viability (Tudor et al. 1999). Similarly, our clonal analysis indicates a requirement of $A T R A P 80$ for cell viability in Drosophila, in agreement with a general role in transcription. In marked contrast, disruption of murine TRAP220 shows that this gene is essential for normal development, but lethality is associated with a complex variety of defects in several tissues and is not cell lethal (Ito et al. 2000). Similarly, the analysis of pap activity suggests a much more specific and restricted role for this gene. No effect on larval cell proliferation was detected, and the tissue-specific consequences of pap mutations for adult cells range from no effect to cell or segment identity transformations.

The large size of known complexes, and the functional diversity observed for different yeast Srb subunits (Holstege et al. 1998), suggest their aptitude to receive and integrate multiple cellular inputs. A previous study of the C. elegans sur-2 gene focused on its role as a genetic suppressor of a Ras/MAP kinase pathway in vulval development, indicating a functional connection between cell signaling and mediator function (Singh and Han 1995). The human TRAP and DRIP complexes were identified by their biochemical interactions with thyroid and vitamin $\mathrm{D}_{3}$ nuclear hormone receptors, and a different subunit, human TRAP220, mediates ligand-specific complex binding to several nuclear receptors (Yuan et al. 1998). The heightened affinity of this specific ligandbound complex and its enhancement of in vitro transcription, in contrast to the nuclear receptor-TRAP220 couple, seems likely to represent a recurrent theme for MED utilization in the dynamic expression of genetic programs during development.

This diversity underlines the need for detailed in vivo elucidation of the roles of different mediator proteins. Are all MED protein functions effected within the framework of a single complex, or can these proteins also participate in other biochemical roles? The clonal analysis for pap indicates a marked discordance between spatial gene expression and gene activity. These observations may represent evidence for positional information inter- 
Fly mediator genes in cell and segment identity

preted through a mediator complex, which is in accord with a previous proposal that MED complexes may act as a sort of molecular control panel (Hampsey and Reinberg 1999). It will be important to better understand the rules of in vivo mediator protein function, notably in transcriptional initiation where potential control points include complex assembly, nuclear localization of subunits or complexes, and posttranslational modifications such as phosphorylation by protein kinases (Singh and Han 1995; Jiang et al. 1998; Boyer et al. 1999) as elements permitting the rapid integration of highly specific spatial information from the cellular environment.

Most descriptions ascribe a coactivator role to MED function. Interestingly, though, observations in vitro have also identified specific subcomplexes that serve as transcriptional corepressors (Song and Carlson 1998; Sun et al. 1998; Balciunas et al. 1999|. Our genetic tests identify pap as a functional antagonist of Scr, whereas tests of molecular epistasis indicate that these genes act in parallel. The observed antagonism with Scr could result indirectly, reflecting the complex circuitry of the genetic program. However, dose-sensitive synergistic functional interactions such as those detected here may also indicate molecular interactions-which are direct or nearly so-through the intermediary of an as yet unidentified homeotic cofactor. Scr is expressed and required throughout the $\mathrm{T} 1$ and labial tissues. However, this requirement is not uniform: SCR-expressing cells give rise to the diverse cell types composing an adult segment. The antagonistic effect of pap on Scr function described here is position-specific within the $\mathrm{T} 1 \mathrm{leg}$, apparently limited to cells yielding the distal sex comb. These apparently paradoxical observations might reflect a crucial role of the MED complex in integrating diverse inputs. In this manner, the localized effect that appears as a negative regulation of Scr might reflect rather the de-repression of a target gene coregulated by Scr with other specific factors. A MED function contributing to a combinatorial output that integrates homeotic input with those of other specific transcription factors would be a coherent role for segmental differentiation.

In a study of the roles of Srb subunits in the yeast gene expression program, extensive functional diversity was observed among the subunits (Holstege et al. 1998). In the present initial analysis of two $M E D$ genes in Drosophila, functional overlap but also clear differences were noted between $d T R A P 80$ and pap. The identification of the $A M E D$ genes should now facilitate the identification of their mutations toward the detailed in vivo characterization necessary for comprehending how the diversity of individual subunit functions can be integrated in a working machine with an important developmental role. The convergence of data from studies of related complexes in yeast, mammals, C. elegans, and now Drosophila should soon afford a clearer picture of how mediators mediate transcriptional regulation throughout development and evolution.

\section{Materials and methods}

Fly techniques

Standard Drosophila culture media and culture methods were employed.

\section{Isolation of pap mutations}

The HSPB transgenic element induces ectopic distal sex combs at low frequency. Among 5000 new autosomal insertions generated by mobilization of an X-linked P-lacW element (http:// flybase.bio.indiana.edu//, the pap $^{1}$ allele (for poils aux pattes, "hairy legs") enhanced distal sex comb frequency in combination with the HSPB:4d line. Phenotypic revertants and imprecise excisions were generated by remobilizing the pap $^{1}$ insertion element with $\Delta 2-3$ transposase from a stable $\Delta 2-3$ chromosomal insertion (http://flybase.bio.indiana.edu/).

\section{In situ hybridization}

Antisense probes were generated by in vitro transcription from linearized plasmids, using T3 or T7 RNA polymerases in the presence of digoxygenin-UTP (Boehringer Mannheim). Probe specificity was verified by hybridization to embryos or larvae with localized overexpression of the corresponding mRNA (engrailed-GAL4/UAS-pap or UAS-TRAP80).

\section{Identification of the dTRAP80 ${ }^{1}$ allele}

This allele, previously referred to as 1/3)s2956 (Spradling et al. 1999), was identified by adjacent sequences located within dTRAP80 coding sequences.

\section{Clonal analysis}

Mitotic recombination experiments were performed using the FLP/FRT recombination system (Xu and Rubin 1993). To examine pap function, we employed the strong or null pap ${ }^{53}$ allele that deletes the putative initiator ATG. For adult effects, y $W$ hsFLP; mwh jv pap ${ }^{53}$ FRT-2A/TM3, Sb females were crossed with $y w ; \mathrm{Dp}(1 ; 3) s c^{S 4} \mathrm{y}^{+} M(3) i^{55}$ FRT-2A/TM3, Sb males. FLP recombinase was induced by heat shock in first- or second-instar larvae $\left(1 \mathrm{~h}, 37^{\circ} \mathrm{C}\right) . S b^{+}$adults were examined for pap Minute $^{+}$clones, using the associated cell-autonomous markers $y, m w h$, and $j v$. For twin spot analysis, pap $^{-}$clones were induced and examined in imaginal discs using the cell autonomous GFP marker expressed from the ubiquitin-63E (UB) promoter (Lee et al. 1988). For these experiments, $y w$ hsFLP; $m w h$ jv pap $^{53}$ FRT-2A/TM6B, Hu Tb females were crossed with $W$; UB-GFP FRT-2A males. For analysis of $S c r$ mutant clones, we employed a chromosome carrying the FRT-82B insertion and the null $S c r^{1}$ allele (kindly provided by A. Percival-Smith) and a chromosome carrying FRT-82B with UB-GFP (3R; obtained from the Indiana University Drosophila Stock Center). Clones were induced by heat shock $\left(1 \mathrm{~h}, 37^{\circ} \mathrm{C}\right)$ during first or second instar, and discs prepared from late L3 larvae. Fixation was for $20 \mathrm{~min}, 22^{\circ} \mathrm{C}$ with $4 \%$ paraformaldehyde. GFP expression was analyzed by confocal microscopy.

\section{DNA techniques}

Standard techniques were employed for cloning, blotting, and sequence analyses.

Cloning and structure of pap

A 9.5-kb EcoRI genomic DNA fragment $3^{\prime}$ to the pap ${ }^{1}$ insertion site cloned by plasmid rescue was used to initiate the isolation 
of $50 \mathrm{~kb}$ of genomic DNA encompassing pap and to probe cDNA libraries. The intron-exon structure of pap/dTRAP240 was determined by comparison of genomic and cDNA sequences. For transgenic rescue, a pap minigene was constructed containing the ubiquitin promoter and a fusion of pap cDNA (the 5' exons; see Fig. 1a) and genomic ( $3^{\prime}$ exons) sequences, and transgenic lines were established following microinjection according to standard methods (Cribbs et al. 1995). GenBank database searches used the BLAST program (http://www.ncbi.nlm.nih. gov/BLAST//. Protein sequence alignments were performed using Clustal W software. Details are available on request.

\section{Structure of dTRAP80}

The intron-exon structure of $d T R A P 80$ was determined by comparison of genomic and cDNA (clone SD10038 from BDGP) sequences. Genbank data base searches and protein sequence alignments were as above.

\section{Localization of dMED homologs}

Listed below are the identified Drosophila genes corresponding to known human mediator proteins (see Fig. 2a), followed by their GadFly identifiers: dARC105 (CG4184); dTRAP26/ TRFP (CG18267), dARC32 (CG13867); dTRAP95 (CG5465); dTRAP150ß/SUR2 (CG3695); dTRAP170/RGR1 (CG12031); dTRAP36 (CG8609); dTRAP100 (CG7999); dTRAP56/CDK8 (CG10572); dTRAP15/NUT2 (CG5057); dTRAP230 (CG8491); pap/dTRAP240 (CG9936); dTRAP220 (CG7162); dTRAP18/ SOH1 (CG1057); dTRAP37 (CG1245); dTRAP32/MED6 (CG9473); dTRAP34/MED7 (CG6529); dTRAP33/cycC/SRB11 (CG7281); dTRAP80/SRB4 (CG7957); dARC42 (CG4703); $d A R C 92$ (CG12254). $d A R C 70$ (CG1793) is situated on the small chromosome IV (not shown), and dTRAP19/SRB7 (CG17397) could not yet be mapped.

\section{Generation of anti-PAP antiserum}

Recombinant hexahistidine-Pap fusion protein including the first 366 amino acid residues of Pap protein was used to immunize rabbits (Eurogentec SA). Recombinant GST-Pap fusion protein blotted to a nitrocellulose filter permitted affinity purification of anti-Pap antisera. Immunostainings employed commercially available reagents. Details are available on request.

\section{Acknowledgments}

This manuscript was much improved through the comments of J. Casanova, G. Giménez, S. González, C. Benassayag, M. Crozatier, B. Glise, and A. Vincent, whom we thank for their critical reading. We thank the Indiana University Drosophila Stock Center and S. Kerridge, A. Percival-Smith, and N. Perrimon for fly stocks; W. Gehring for the anti-Scr serum; P. Cochard for help with the confocal microscope; and A. Monier and A. Lepage for expert technical help. We also acknowledge the contributions of A. Roques, Sé. Peyrefitte, and E. Vanrobays to the structural characterization of pap. This work benefited from the ongoing support of the Centre National de Recherche Scientifique (CNRS) and grants from the Association pour la Recherche sur le Cancer (ARC) and from the MinistÈre de 1'Education Supérieure et la Recherche (MESR) of France. M.B. was financed by graduate fellowships from the MESR and the ARC.

The publication costs of this article were defrayed in part by payment of page charges. This article must therefore be hereby marked "advertisement" in accordance with 18 USC section 1734 solely to indicate this fact.

\section{References}

Adams, M.D., Celniker, S.E., Holt, R.A., Evans, C.A., Gocayne, J.D., Amanatides, P.G., Scherer, S.E., Li, P.W., Hoskins, R.A., Galle, R.F., et al. 2000. The genome sequence of Drosophila melanogaster. Science 287: 2185-2195.

Asturias, F.J., Jiang, Y.W., Myers, L.C., Gustafsson, C.M., and Kornberg, R.D. 1999. Conserved structures of mediator and RNA polymerase II holoenzyme. Science 283: 985-987.

Balciunas, D., Galman, C., Ronne, H., and Bjorklund, S. 1999. The Med 1 subunit of the yeast mediator complex is involved in both transcriptional activation and repression. Proc. Natl. Acad. Sci. 96: 376-381.

Boube, M., Benassayag, C., Seroude, L., and Cribbs, D.L. 1997. Ras1-mediated modulation of Drosophila homeotic function in cell and segment identity. Genetics 146: 619-628.

Boyer, T.G., Martin, M.E., Lees, E., Ricciardi, R.P., and Berk, A.J. 1999. Mammalian Srb/Mediator complex is targeted by adenovirus E1A protein. Nature 399: 276-279.

Chao, D.M., Gadbois, E.L., Murray, P.J., Anderson, S.F., Sonu, M.S., Parvin, J.D., and Young, R.A. 1996. A mammalian SRB protein associated with an RNA polymerase II holoenzyme. Nature 380: 82-85.

Cribbs, D.L., Benassayag, C., Randazzo, F.M., and Kaufman, T.C. 1995. Levels of homeotic protein function can determine developmental identity: Evidence from low-level expression of the Drosophila homeotic gene proboscipedia under Hsp70 control. EMBO J. 14: 767-778.

Gu, W., Malik, S., Ito, M., Yuan, C.X., Fondell, J.D., Zhang, X., Martinez, E., Qin, J., and Roeder, R.G. 1999. A novel human SRB/MED-containing cofactor complex, SMCC, involved in transcription regulation. Mol. Cell 3: 97-108.

Hampsey, M. and Reinberg, D. 1999. RNA polymerase II as a control panel for multiple coactivator complexes. Curr. Opin. Genet. Dev. 9: 132-139.

Holstege, F.C., Jennings, E.G., Wyrick, J.J., Lee, T.I., Hengartner, C.J., Green, M.R., Golub, T.R., Lander, E.S., and Young, R.A. 1998. Dissecting the regulatory circuitry of a eukaryotic genome. Cell 95: 717-728.

Ito, M., Yuan, C.X., Malik, S., Gu, W., Fondell, J.D., Yamamura, S., Fu, Z.Y., Zhang, X., Qin, J., and Roeder, R.G. 1999. Identity between TRAP and SMCC complexes indicates novel pathways for the function of nuclear receptors and diverse mammalian activators. Mol. Cell 3: 361-370.

Ito, M., Yuan, C., Okano, H., Darnell, R., and Roeder, R. 2000. Involvement of the TRAP220 component of the TRAP/ SMCC coactivator complex in embryonic development and thyroid hormone action. Mol. Cell 5: 683-693.

Jiang, Y.W., Veschambre, P., Erdjument-Bromage, H., Tempst, P., Conaway, J.W., Conaway, R.C., and Kornberg, R.D. 1998. Mammalian mediator of transcriptional regulation and its possible role as an end-point of signal transduction pathways. Proc. Nat1. Acad. Sci. 95: 8538-8543.

Kornberg, R.D. 1999. Eukaryotic transcriptional control. Trends Cell Biol. 9: M46-M49.

Kwon, J.Y., J.M. Park, B.S. Gim, S.J. Han, J. Lee, and Y.J. Kim. 1999. Caenorhabditis elegans mediator complexes are required for developmental-specific transcriptional activation. Proc. Nat1. Acad. Sci. 96: 14990-14995.

Lee, H.S., Simon, J.A., and Lis, J.T. 1988. Structure and expression of ubiquitin genes of Drosophila melanogaster. Mol. Cell. Biol. 8: 4727-4735. 
Lee, T.I., Wyrick, J.J., Koh, S.S., Jennings, E.G., Gadbois, E.L., and Young, R.A. 1998. Interplay of positive and negative regulators in transcription initiation by RNA polymerase II holoenzyme. Mol. Cell. Biol. 18: 4455-4462.

Lee, Y.C. and Kim, Y.J. 1998. Requirement for a functional interaction between mediator components Med6 and Srb4 in RNA polymerase II transcription. Mol. Cell. Biol. 18: 53645370.

Lee, Y.C., Park, J.M., Min, S., Han, S.J., and Kim, Y.J. 1999. An activator binding module of yeast RNA polymerase II holoenzyme. Mol. Cell. Biol. 19: 2967-2976.

Malik, S. and Roeder, R. 2000. Transcriptional regulation through Mediator-like coactivators in yeast and metazoan cells. Trends Biochem. Sci. 25: 277-283.

Naar, A.M., Beaurang, P.A., Robinson, K.M., Oliner, J.D., Avizonis, D., Scheek, S., Zwicker, J., Kadonaga, J.T., and Tjian, R. 1998. Chromatin, TAFs, and a novel multiprotein coactivator are required for synergistic activation by $\mathrm{Sp} 1$ and SREBP-1a in vitro. Genes \& Dev. 12: 3020-3031.

Naar, A.M., Beaurang, P.A., Zhou, S., Abraham, S., Solomon, W., and Tjian, R. 1999. Composite co-activator ARC mediates chromatin-directed transcriptional activation. Nature 398: 828-832.

Parvin, J.D. and Young, R.A. 1998. Regulatory targets in the RNA polymerase II holoenzyme. Curr. Opin. Genet. Dev. 8: $565-570$.

Pattatucci, A.M., Otteson, D.C., and Kaufman, T.C. 1991. A functional and structural analysis of the Sex combs reduced locus of Drosophila melanogaster. Genetics 129: 423-441.

Percival-Smith, A., Weber, J., Gilfoyle, E., and Wilson, P. 1997. Genetic characterization of the role of the two HOX proteins, Proboscipedia and Sex Combs Reduced, in determination of adult antennal, tarsal, maxillary palp and proboscis identities in Drosophila melanogaster. Development 124: 5049-5062.

Rachez, C., Lemon, B.D., Suldan, Z., Bromleigh, V., Gamble, M., Naar, A.M., Erdjument-Bromage, H., Tempst, P., and Freedman, L.P. 1999. Ligand-dependent transcription activation by nuclear receptors requires the DRIP complex. Nature 398: 824-828.

Rubin, G.M., Hong, L., Brokstein, P., Evans-Holm, M., Frise, E., Stapleton, M., and Harvey, D.A. 2000. A Drosophila complementary DNA resource. Science 287: 2222-2224.

Ryu, S., Zhou, S., Ladurner, A.G., and Tjian, R. 1999. The transcriptional cofactor complex CRSP is required for activity of the enhancer-binding protein Sp1. Nature 397: 446-450.

Singh, N. and Han, M. 1995. sur-2, a novel gene, functions late in the let-60 ras-mediated signaling pathway during Caenorhabditis elegans vulval induction. Genes \& Dev. 9: 2251-2265.

Song, W. and Carlson, M. 1998. Srb/mediator proteins interact functionally and physically with transcriptional repressor Sfl1. EMBO J. 17: 5757-5765.

Spahr, H., Beve, J., Larsson, T., Bergstrom, J., Karlsson, K.A., and Gustafsson, C.M. 2000. Purification and characterization of RNA polymerase II holoenzyme from Schizosaccharomyces pombe. J. Biol. Chem. 275: 1351-1356.

Sun, X., Zhang, Y., Cho, H., Rickert, P., Lees, E., Lane, W., and Reinberg, D. 1998. NAT, a human complex containing Srb polypeptides that functions as a negative regulator of activated transcription. Mol. Cell 2: 213-222.

Taylor, W.R. 1986. Identification of protein sequence homology by consensus template alignment. J. Mol. Biol. 188: 233-258.

Thompson, C.M., Koleske, A.J., Chao, D.M., and Young, R.A. 1993. A multisubunit complex associated with the RNA polymerase II CTD and TATA-binding protein in yeast. Cell
73: $1361-1375$.

Tudor, M., Murray, P.J., Onufryk, C., Jaenisch, R., and Young, R.A. 1999. Ubiquitous expression and embryonic requirement for RNA polymerase II coactivator subunit $\mathrm{Srb} 7$ in mice. Genes \& Dev. 13: 2365-2368.

$\mathrm{Xu}, \mathrm{T}$. and Rubin, G.M. 1993. Analysis of genetic mosaics in developing and adult Drosophila tissues. Development 117: 1223-1237.

Yuan, C.X., Ito, M., Fondell, J.D., Fu, Z.Y., and Roeder, R.G. 1998. The TRAP220 component of a thyroid hormone receptor-associated protein (TRAP) coactivator complex interacts directly with nuclear receptors in a ligand-dependent fashion. Proc. Natl. Acad. Sci. 95: 7939-7944. 


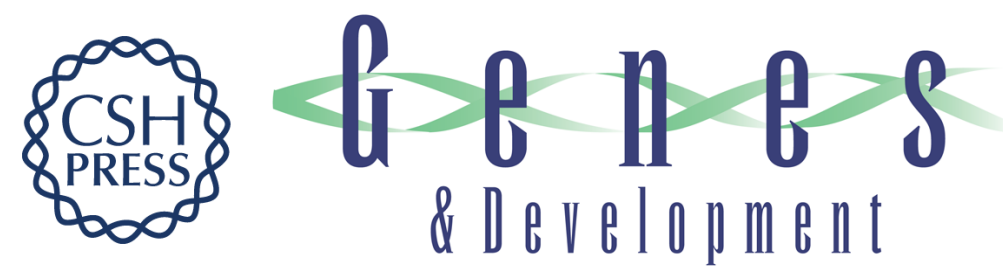

\section{Drosophila homologs of transcriptional mediator complex subunits are required for adult cell and segment identity specification}

Muriel Boube, Christian Faucher, Laurent Joulia, et al.

Genes Dev. 2000, 14:

Access the most recent version at doi:10.1101/gad.17900

References This article cites 37 articles, 20 of which can be accessed free at: http://genesdev.cshlp.org/content/14/22/2906.full.html\#ref-list-1

License

Email Alerting

Receive free email alerts when new articles cite this article - sign up in the box at the top Service right corner of the article or click here.

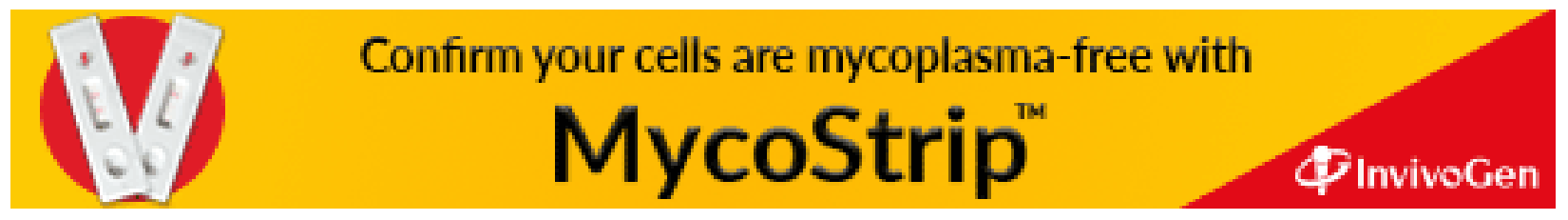

